\title{
Bypassing primary healthcare institutions: Reasons identified by patients' attending the out-patient department
}

\author{
Karunaratne NP ${ }^{1}$, Kumara GSP ${ }^{1}$, Karunathilake KTGS $^{1}$, Karunathilake GVKM $^{1}$, Kaushalya PGM ${ }^{1}$, \\ Kavinda $\mathrm{HWI}^{1}$, Keshala AAM ${ }^{1}$, Ponnamperuma $\mathrm{T}^{2}$
}

1. Medical Undergraduate, Faculty of Medicine, University of Ruhuna

2. Senior Lecturer, Department of Community Medicine, Faculty of Medicine, University of Ruhuna

\section{Corresponding Author:}

Thyagi Ponnamperuma, Department of Community Medicine, Faculty of Medicine, University of Ruhuna

https://orcid.org/0000-0002-0031-3483

\begin{abstract}
Introduction

Sri Lanka has a hierarchy of health services, from health care units with fewer facilities to hospitals with specialized care. Due to the absence of well demarcated draining areas and lack of clear referral policies, patients tend to bypass the primary health care units and attend much superior health care institutions without referrals. This practice has caused congestion in hospitals and deterioration of efficacy and quality of care provided to needy patients.
\end{abstract}

This study was designed to identify the reasons for bypassing primary health care institutions by patients.

\section{Method}

A cross sectional study was done among conveniently selected sample of patients attending outpatient department of Teaching Hospital Karapitiya (THK). Data were collected by an interviewer administered questionnaire that assessed their socio demographic status and reasons for bypassing their nearest hospital. Patients whose nearest hospital are THK and referred patients were excluded from the study.

\section{Results}

Among 344 patients, $60.2 \%$ were females, mean age was 41 years. Most were educated up to GCE O/L, unemployed, and had an average income $<$ Rs 30,000 . More than half $(52 \%)$ of the patients had a primary health care unit less than $5 \mathrm{~km}$ from their residence. In average, patients spent nearly $1 \& 1 / 2$ hours for traveling and paid Rs. 186 to reach tertiary care hospital. According to the participants' understanding, most of their nearest hospitals had adequate supply of medicine (72\%) and staff $(79 \%)$, but not the laboratory facilities (48\%). Poor quality of service represented by of doctors, delayed service, lack of essential facilities and lack of laboratory facilities and specialized services were reported as main reasons for by passing their nearest health care institutions.

\section{Conclusions}

Findings confirmed that averagely educated people with low middle-income avoid primary health care services due to unavailability of specialized care, inadequate facilities and poor quality of service. 
Improving facilities and building trust on primary care service will therefore improve utilization of primary health care institutions in the country.

\section{Introduction}

Sri Lanka's health system has achieved indicators of health status and service coverage well above the

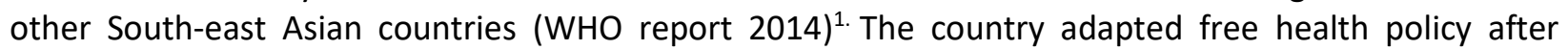
gaining the independence and the health service developed thereafter. The ministry of health and indigenous medicine is the key responsible institution in providing health services and its goals to provide sufficient quality of health service in preventive, curative, rehabilitative and palliative care to all people in the country. A majority of the population has easy access to a reasonable level of health care facilities and free government health care is available within $4.8 \mathrm{Km}$ of residence. ${ }^{2}$ By the end of 2016, there were 629 curative care hospitals and 480 primary medical care units under the ministry of health. Further, there are 89.5 doctors per 100,000 patients. $^{3}$

The curative health care service possesses an extensive network of health care institutions. These institutions are categorized in a hierarchy based on available facilities; hospitals with specialized care to health care units with fewer facilities. They are; National Hospital, Teaching Hospitals, Provincial General Hospitals, District General Hospitals, Base Hospital Type-A, Base Hospital Type-B, Divisional Hospital Type-A, Divisional Hospital Type-B, Divisional Hospital Type-C and Primary Medical Care Units. Specialized care is provided through Base, District General, Provincial General, Teaching and some selected specialized hospitals. With few exceptions, the divisional hospitals and all the primary medical care units are served by non-specialist medical officers.

Even though the country established a remarkable health care delivery system, only recently the Ministry of Health streamline the referrals among primary health care services and from primary to secondary to tertiary health care services. According to this, a patient should be first seen by a medical officer at their nearest primary care institution, and if needed refer them to the nearest secondary care hospital for further treatment. This procedure makes health service to function more efficiently and effectively. However, the health care system has not yet fully adapted this referral policy and this allowed patients to bypass primary health care units and attend much superior health care institutions even for minor illnesses without referral.

Bypassing primary health care leads to the underutilization of primary health care facilities and to the overutilization of secondary and tertiary care facilities. With underutilization of primary care institutions, allocated resources get wasted and the performance of medical staff gets impaired. Meanwhile, increase utilization of secondary and tertiary care institutions by patients with minor ailments causes congestion in these institutions and deterioration of quality of service provided for needy patients. ${ }^{4}$ To find solutions to this problem, it is necessary to identify the reasons for not utilizing primary care institutions by patients in the country. This study was thus designed to identify the reasons for bypassing primary health care by patients attending the main teaching hospital in the South of Sri Lanka.

\section{Methods}

Teaching Hospital Karapitiya (THK) is the only tertiary care hospital in the south of the country, which is located in Galle district. Every day, approximately 1000 patients get treatment from the outpatient department in this hospital. Present study was conducted among conveniently selected sampleof 344 patients who attended the outpatient department of THK. Data were collected on 5 different days within 2 weeks duration from 8am.to 12 noon in February 2019. All the patients above 18 years of age, 
and persons accompany inpatients below age 18 years of age were interviewed. We excluded all the patients whose nearest hospital is THK.

Patients were subjected to a interviewer-administered questionnaire designed by the research team and pre-tested at the out-patient department in the General Hospital Matara. The questionnaire consisted with six sections; 1. Socio-demographic information, 2. Information about the nearest hospital, 3. Reasons for bypassing the nearest hospital, 4. Reasons for attending THK, 5. Information on travel time and cost6. Presenting complain of the patient. Research group members conducted the interviews. Data were analyzed by SPSS statistical software version 25. Descriptive statistics were use in this study.

Ethical clearance was obtained by the ethical review committee, Faculty of Medicine, University of Ruhuna. Permission was obtained by the director of Teaching Hospital Karapitiya. Informed consent was obtained from all participants of the study.

\section{Results}

There were 344 patients with $60 \%$ females. The mean age of the patients was 40.9 years (SD $=18.2$, range $1-85)$. There were $13.4 \%$ of patients under 18 years of age and the highest number was reported in the age category $36-60$ years (46\%). Majority was Sinhalese $(96.2 \%)$, and Tamils(0.9\%) and Muslims $(2.9 \%)$ were reported in a lesser number. Most of the patients over 18 years were married $(88 \%)$ and had studies up to GCE O/L (34\%) while $8.1 \%$ had completed higher education. Above the age of 18 years, many were unemployed $(35.8 \%)$ and the others were self-employed $(27.3 \%)$ or worked in the private sector $(23.5 \%)$ or in the government sector (13.4\%). A majority(67.2\%).of the patients had a monthly income fewer than 30000 rupees (mean 29003 rupees).

As expected, most of the patients were from Galle district (73.7\%) while the others were from Matara $(18.6 \%)$, Hambantota $(4.9 \%)$, Ratnapura (2\%), Monaragala $(0.3 \%)$ and Kalutara districts (0.6\%). We excluded all patients who were not residents of the Galle district and were referred to THK and then inquired about their nearest hospital to their residence. Closest hospital was a base hospital in $21.0 \%$, district hospitals in $26.4 \%$, divisional hospitals in $27.0 \%$, PMCU in $10.4 \%$ and a rural hospital in $3.2 \%$ of patients. Most of the patients had come by public transport (76.5\%). On average, they had spent nearly $1 \& 1 / 2$ hours for traveling and paid Rs. 186 to reach THK. As shown in figure 1 , the three common presentations to OPD were joint pain followed by wounds and urinary symptoms.

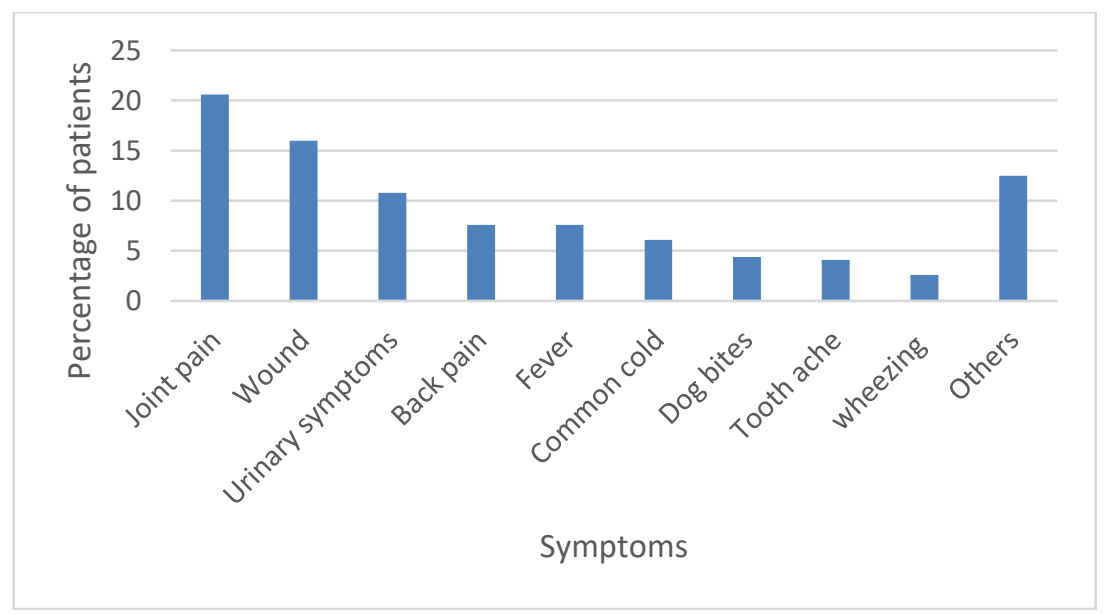

Figure 1. Common presentations to OPD 
Among the subsample from Galle district, $95.6 \%$ had taken treatment from their nearest hospital at least one time in their life, but $4.4 \%$ had never visited these institutions.In $57 \%$ of the patients, the nearest governmenthospitalwas located $<5 \mathrm{~km}$ from theirresidence. They reported that they can reach their nearest hospital less than half an hour (mean $=26$ minutes) spending an average cost of Rs. 42 for their transport.

According to patients and accompanying guardians understanding, their nearest hospitals had adequate facilities. Figure2, shows the percentage of patients who believed that their nearest hospital had adequate staff $77.5 \%$, adequatesupply of medicine $69.6 \%$ and laboratory facilities $41.8 \%$. Further, the participants stated their reasons for not attending their nearest hospital.

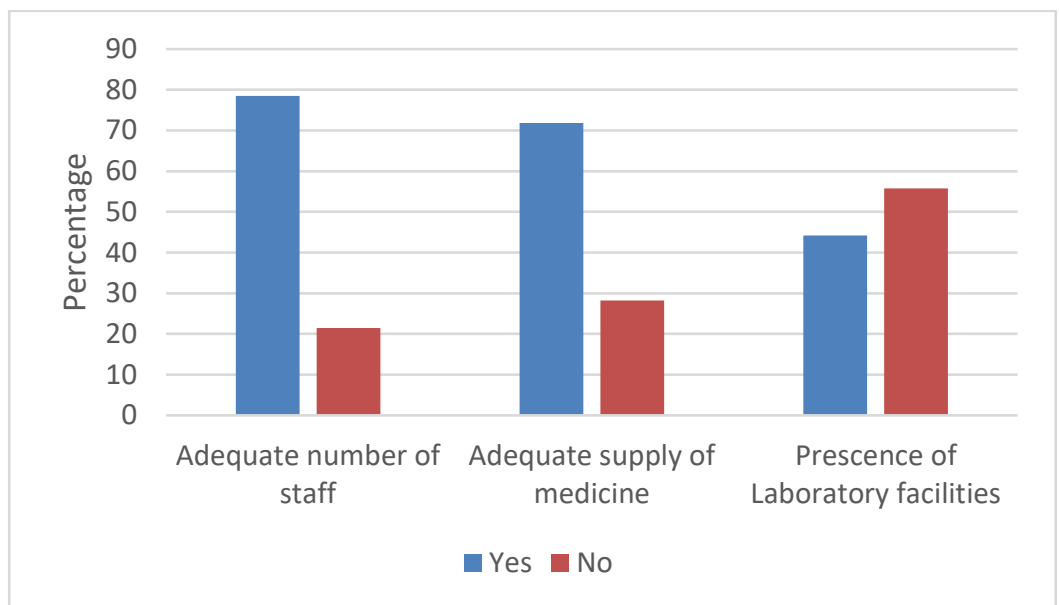

Figure 2: Patient perceptions of the Facilities available at their nearest hospital

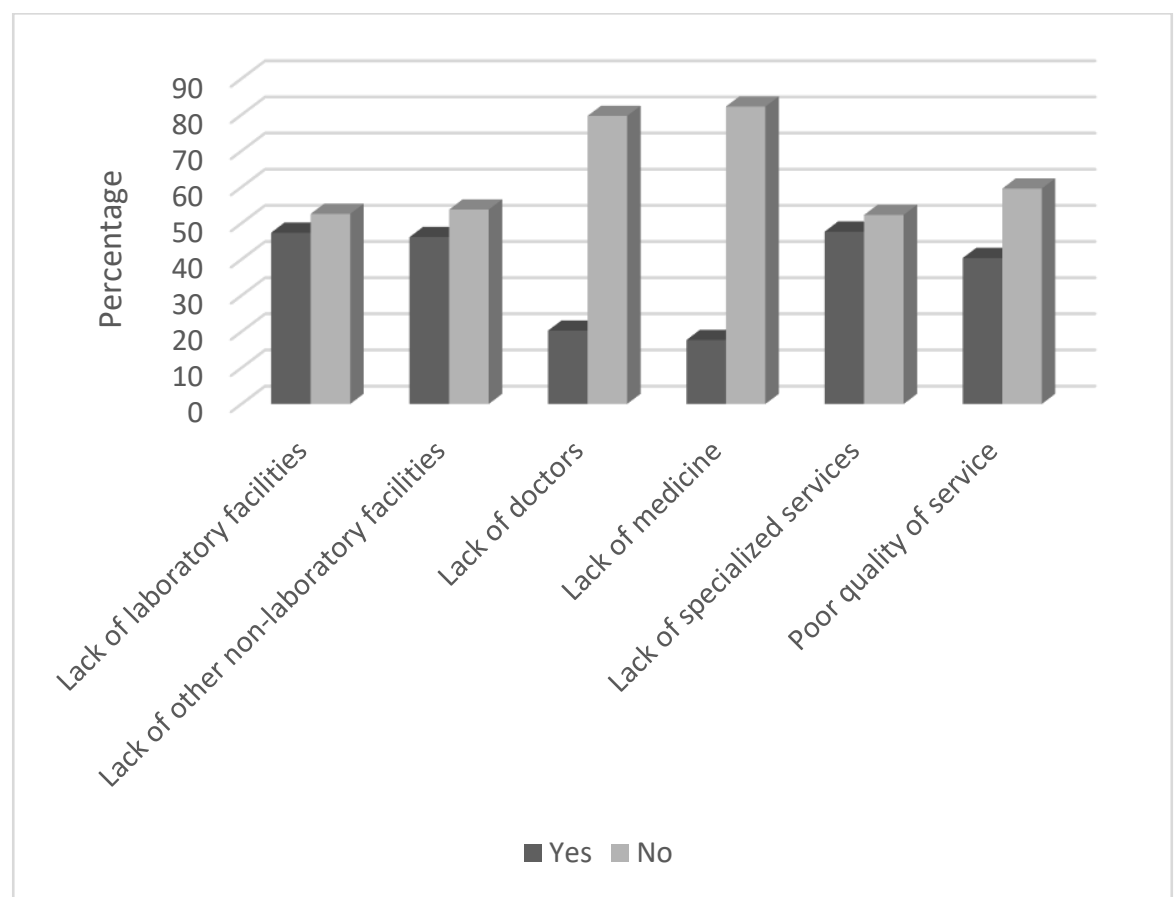

Figure 3. Common reasons stated by patients for not attending the nearest hospital 
As depicted in figure 3, the common reasons they identified were the lack of laboratory facilities and other non-laboratory facilities, followed by the absence of specialized care and poor quality of service.

Poor quality of service provided by doctors, delays in service, lack of laboratory facilities and specialized services were reported as main reasons for by passing their nearest health care institutions. Patients who were residing outside Galle district had visited the OPDas referred patients or to take treatment while they were visiting an inward relative at THK.

\section{Discussion}

This study explored the patient characteristics and reasons for by passing primary health care institutions among patients attending the outpatient department at Teaching Hospital Karapitiya. It was observed that most middle-aged, married females with low-middle economic status attended OPD of THK by passing primary health care institutions for treatment. Even though we observed that middleaged patients bypassed primary healthcare facilities, some studies have found that older patients were most likely to bypass primary health care than the young, ${ }^{6,7}$ Perera\&Weerasinghe's (2015) study also showed that female over-representation for OPD treatment, where they state that high female unemployment and males being at work during the OPD functioning hours as the reason for this observation. ${ }^{4}$ Similar to our findings, Jiexin et al (2008) found that married patients were more likely to bypass primary health care than single because extended support can be obtained from the spouse or other family members for travel. ${ }^{6}$ A majority of the study participants had an income less than Rs.30,000. According to the household income and expenditure survey (Department of Census and Statistics -2016), the mean income of the income receivers in the country was Rs 33,894. Based on this, we can assume that low-middle income families have bypassed the primary health care institution to attend tertiary care. This is supported by the previous finding that the poor were more likely to bypass primary health care institutions than the others. ${ }^{5}$

Based on our results patients have bypassed their nearest hospital due to three main reasons. One, due to a lack of facilities. A considerable number of patients (25\%) have stated that these hospitals have inadequate doctors and medicine. Also, more than half have said that these hospitals have no laboratory facilities. Previous studies have also found that hospitals with fewer facilities are more likely to be bypassed by patients ${ }^{8,9}$. In this case, improving facilities will improve the utilization of primary care institutions, which is a task to be undertaken by the ministry of health. Second, due to the lack of specialized care. Close to $75 \%$ of the patients have bypassed their nearest hospital even after believing these hospitals have an adequate number of doctors and medicine. The reason they have stated for attending THK was the availability of specialized services. Previous research has also identified that hospitals host specialized care are more likely to be visited by patients to take treatment even for minor ilnesses. ${ }^{10}$ This indicates that patients believe specialized services are needed to treat most of the health problems. In this case, educating the public and changing their attitudes could potentially improve the utilization of primary care institutions. The third reason that patients have identified was the poor quality of service at the peripheral hospitals. Poor quality of service could be due to several reasons including lack of facilities. This study has not obtained detailed information in this aspect which is an identified limitation. Further studies are therefore recommended to address this issue to improve primary health care services. 
Many patients have traveled to THK by public transport. When compared to the meantime spent for travelling and cost; patients have spent Rs186 and $1 \& 1 / 2$ hours to reach THK while it was only Rs.42 and 25 minutes required to go to the nearest hospital. Even with these added costs and extra time, people have bypassed them due to the above-mentioned reasons.

The current study has several limitations. First, although most of the participants stated that their nearest hospital had adequate staff and medicine; a considerable number of patients were living close to secondary care hospitals with relatively more facilities than primary care facilities. A separation of patients based on the category of the nearest hospital with a larger sample size would have clarified the reasons for bypassing in a greater degree. Second, inquiring about hospital facilities in a broad context like in this study would have hindered depicting a clear picture of patients' perception of available facilities that are important to them. Fourth, patients visiting the OPD from outside Galle district would have been excluded at the designer level of the study; unexpectedly, the present study had to leave out $25 \%$ of the sample from the core analysis. Finally, participants' perception on the quality of the service provided by their nearest hospital would have been measured in more detail by an elaborate questionnairespecifying many domains of quality.

In conclusion, we would like to emphasize that averagely educated people with low-to-middle income avoid primary health care services due to lack of facilities and poor quality of service. It is clear from their symptoms, that most of the disease presentations could have been treatedadequately at the primary care setting. Therefore, it is vitally important to educate and buildup the trust of the public on primary healthcare institutions while improving facilities and quality of service in primary care setting.

\section{Acknowledgements:}

We are grateful to Prof. PV De Silva, Dr. Champa Wijesinghe and all the academic staff of the department of Community medicine, University of Ruhuna and all patients who willingly participated in this study

\section{References}

1) Measuring core health indicators in the South-East Asian region, WHO regional office for South-East Asia (http://apps.searo.who.int/).

2) de Silva A, Ranasinghe T, Abeykoon P. Universal health coverage and the health Sustainable Development Goal: achievements and challenges for Sri Lanka. WHO South-East Asia J Public Health 2016; 5(2): 82-88.

3) Annual Health Bulletin.2016(http://www.health.gov.lk/moh final/english/others.php?pid=110)

4) Perera SK, Weerasinghe MC. Bypassing primary care in Sri Lanka: a comparative study on reasons and satisfaction. Vietnam Journal of Public Health, 2015, 3 (1).

5) AkinJ. S, Hutchinson P. Health- care facility choice and phenomenon of bypassing. Health Policy and Planning 1999; 14:135-151.

6) Jiexin L., BellamiG., Barnet B., Weng S. Bypass of Local Primary Care in Rural Counties: Effect of Patient and Community Characteristics. Annals of Family Medicine.2008; 6:124-130.

7) Basu J, Cooper J. Out-of-Area Travel From Rural and Urban Counties: A Study of Ambulatory Care Sensitive Hospitalizations for New York State Residents. The Journal of Rural Health. 2000; 16: 129-138.

8) George G., Sara B., Kent R., David G. Healthcare seeking behavior and out of pocket payments in Tbilisi, Georgia. Health Policy and Planning. 2005; 20(4):131-135. 
9) Margaret E. K., Godfrey M, Colin W. M., Molly M., Peter C. R., Sandro G. Bypassing primary care facilities for childbirth: a population-based study in rural Tanzania. Health Policy and Planning. 2009; 24: 279-288.

10) Kruk M. E., Mbaruku G., McCord C. W., Moran M., Rockers P. C., Galea S. Bypassing primary care facilities for childbirth: a population- based study in rural Tanzania. Health Policy Plan. 2009; 24:27988.

11) Leonard K. L., Mlinga G. R., Mariam D. H. Bypassing health centers in Tanzania: revealed preferences for observable and non-observable quality. Department of economics discussion paper series. 2002; 1-4 